INPLASY

PROTOCOL

To cite: He et al. Efficacy and safety of Tripterygium wilfordii glycosides in treatment of $\lg \mathrm{A}$ nephropathy: A systematic review and meta-analysis. Inplasy protocol 202210037. doi:

10.37766/inplasy2022.1.0037

Received: 09 January 2022

Published: 09 January 2022

Corresponding author:

Mingyu He

ccucmhmy2052@163.com

Author Affiliation:

Changchun University of

Chinese Medicine

Support: National Natural

Science Found.

Review Stage at time of this submission: Data analysis.

Conflicts of interest:

None declared.

\section{Efficacy and safety of Tripterygium wilfordii glycosides in treatment of IgA nephropathy: A systematic review and meta-analysis}

\author{
He, $\mathrm{M}^{1}$; Chang, T2; Zhang, $\mathrm{S}^{3}$.
}

Review question / Objective: The purpose of this randomized controlled trial is to analyze the clinical efficacy and safety of Tripterygium wilfordii polyglycoside and conventional basic treatment in patients with IgA nephropathy, so as to guide clinical rational drug use.

Condition being studied: Efficacy and safety of Tripterygium wilfordii glycosides in treatment of IgA nephropathy. Data have been extracted and analyzed under the guidance of professionals; The team members are all doctors and masters; Team members have visiting experience in the United States.

Information sources: We will search for articles from several electronic databases, including PubMed, EMBASE and the Cochrane Library, in English. Chinese databases include cnKI, Wanfang database, VIP database and CBM. Keywords of the search mainly included: Tripterygium wilfordii polyglycosides, IgA nephropathy, randomized control.

INPLASY registration number: This protocol was registered with the International Platform of Registered Systematic Review and Meta-Analysis Protocols (INPLASY) on 09 January 2022 and was last updated on 09 January 2022 (registration number INPLASY202210037).

\section{INTRODUCTION}

Review question / Objective: The purpose of this randomized controlled trial is to analyze the clinical efficacy and safety of Tripterygium wilfordii polyglycoside and conventional basic treatment in patients with IgA nephropathy, so as to guide clinical rational drug use.

Condition being studied: Efficacy and safety of Tripterygium wilfordii glycosides in treatment of IgA nephropathy. Data have been extracted and analyzed under the 
guidance of professionals; The team members are all doctors and masters; Team members have visiting experience in the United States.

\section{METHODS}

Participant or population: IgA nephrosis patients.

Intervention: Tripterygium wilfordii Hook F.

Comparator: ACEI/ARB; MMF; Hormones and immunosuppressants.

Study designs to be included: RCT.

Eligibility criteria: Kdigo guidelines.

Information sources: We will search for articles from several electronic databases, including PubMed, EMBASE and the Cochrane Library, in English. Chinese databases include cnKI, Wanfang database, VIP database and CBM. Keywords of the search mainly included: Tripterygium wilfordii polyglycosides, IgA nephropathy, randomized control.

Main outcome(s): Efficacy and safety.

Quality assessment / Risk of bias analysis: Cochrane.

Strategy of data synthesis: Revman software was selected for data analysis, random effect size combination was selected for data with heterogeneity, and subgroup analysis was conducted.

Subgroup analysis: Depending on the course of the disease.

Sensitivity analysis: Revman.

Keywords: Tripterygium wilfordii; iga nephropathy; RCT.

Contributions of each author:

Author 1 - Mingyu He.

Author 2 - Tianying Chang.

Author 3 - Shoulin Zhang. 